\title{
Writing Impairments in Japanese Patients with Mild Cognitive Impairment and with Mild Alzheimer's Disease
}

\author{
Atsuko Hayashi ${ }^{a}$ Hiroshi Nomura ${ }^{b}$ Ruriko Mochizuki ${ }^{b}$ \\ Ayumu Ohnuma $^{\text {b }}$ Teiko Kimparab ${ }^{b}$ Kyoko Suzuki ${ }^{d}$ Etsuro Moric \\ a Department of Rehabilitation Science, Kobe University Graduate School of Health Sciences,

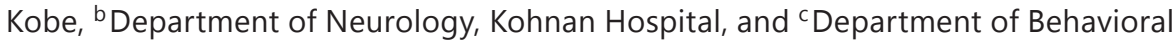 \\ Neurology and Cognitive Neuroscience, Tohoku University Graduate School of Medicine, \\ Sendai, and d Department of Clinical Neuroscience, Yamagata University Graduate School of \\ Medicine, Yamagata, Japan
}

\section{Key Words}

Dementia $\cdot$ Mild cognitive impairment $\cdot$ Kanji $\cdot$ Kana $\cdot$ Narrative writing $\cdot$ Writing disorder

\section{Abstract}

Background/Aims: We investigated writing abilities in patients with the amnestic type of mild cognitive impairment (aMCI) and mild Alzheimer's disease (AD). To examine the earliest changes in writing function, we used writing tests for both words and sentences with different types of Japanese characters (Hiragana, Katakana, and Kanji). Methods: A total of 25 aMCI patients, 38 AD patients, and 22 healthy controls performed writing to dictation for Kana and Kanji words, copied Kanji words, and wrote in response to a picture story task. Analysis of variance was used to test the subject group effects on the scores in the above writing tasks. Results: For the written Kanji words, the mild AD group performed worse than the aMCI group and the controls, but there was no difference between the aMCI group and the controls. For the picture story writing task, the mild $A D$ and aMCI groups performed worse than the controls, but the difference between the $\mathrm{AD}$ and the $\mathrm{aMCI}$ groups was not significant. Conclusions: The mild AD group showed defects in writing Kanji characters, and the aMCI group showed impairments in narrative writing. Our study suggests that narrative writing, which demands complex integration of multiple cognitive functions, can be used to detect the subtle writing deficits in aMCI patients.

(C) 2015 S. Karger AG, Basel 


\section{Introduction}

The development of cholinergic therapies for Alzheimer's disease (AD) has emphasized the importance of early diagnosis and fueled an interest in mild cognitive impairment (MCI). This is an intermediate and transitional stage between normal brain aging and dementia and is regarded as the predementia prodrome of $\mathrm{AD}$ [1]. $\mathrm{MCI}$ is also referred to as minimal $\mathrm{AD}$, questionable $\mathrm{AD}$, or isolated memory deficit. Patients with $\mathrm{MCI}$ present with memory complaints and perform below the norms for their age and education on neuropsychological memory tasks but have relatively normal general cognitive abilities, maintain activities of daily living, and do not have dementia [2].

These criteria require that the general cognitive function is preserved, but the possibility that patients with MCI may have dysfunctions in multiple cognitive domains has been recognized [3]. Ribeiro et al. [3] suggested that patients with MCI frequently exhibit deficits in cognitive domains beyond memory, such as temporal orientation, semantic fluency, language comprehension, calculation, and motor initiative. However, few studies include any assessment of writing impairments. Very mild AD patients with Mini-Mental State Examination (MMSE) scores ranging from 24 to 28 showed mild surface dysgraphia when writing words to dictation, but their writing scores did not differ significantly from those of controls [4]. By contrast, writing impairments manifest in patients with mild AD [5]. Previous studies have suggested that mild AD patients show lexical agraphias, which are characterized by phonologically plausible spelling errors when writing words to dictation (e.g. jealousy $\rightarrow$ gelosy) $[4,6,7]$.

Some studies have shown sentence writing impairments in AD patients using a picture description task comprising simple line drawings [6, 8-12] or a spontaneous writing task [13-15]. In the previous studies that used a picture description task, AD patients wrote shorter sentences [6, 8-11], presented decreased amounts of information $[6,9,10,12]$, and used grammatically simpler sentences relative to controls $[10,12]$. Their writing also showed lexical and semantic errors $[6,8,10,11]$. In the spontaneous writing task, it has been suggested that there were no significant differences between $\mathrm{AD}$ patients and controls in the number of words they used in their sentences. However, as the disease progressed in the AD patients, they showed the following differences: a decreased amount of information [13], grammatically simpler sentences $[13,14]$, more lexical errors, such as allographic types of errors $(a \rightarrow$ A), no punctuation, and spelling errors [15].

The results of the picture description task [12] suggested that patients with minimal AD did not differ significantly from controls in terms of syntactic measures and the number of paraphasias, but the information content presented by patients with minimal AD was worse than that of controls. Very mild AD patients had much more punctuation errors [15] and wrote simpler sentences [13] compared with controls in a spontaneous writing task. These findings suggest that writing tasks might be useful for detecting subtle language deficits in patients with MCI.

In Japan, there have been some studies of writing impairments in AD patients. The Japanese writing system has two types of scripts: Kana (syllabogram) and Kanji (morphogram). Kana characters have simple graphic forms which are similar to those of Western alphabets, and each character has a one-to-one correspondence with a syllable. Kanji characters, by contrast, have complex graphic forms, and they are associated with multiple semantic and phonetic representations. Because of these dual writing systems, agraphia in Japanese patients can have different patterns: Kanji agraphia and Kana agraphia. Kanji agraphia is thought to correspond to lexical agraphia in Western languages. By contrast, Kana agraphia corresponds to phonological agraphia [16]. Hayashi et al. [17], in a cohort study, found that mild AD patients showed difficulties with Kanji writing, particularly many nonresponse errors, although they had no problems writing in Kana and copying Kanji. 
Hayashi et al.: Writing Impairments in Japanese Patients with Mild Cognitive Impairment and with Mild Alzheimer's Disease

Table 1. Subject characteristics

\begin{tabular}{lcccr}
\hline & $\begin{array}{l}\text { Controls } \\
(\mathrm{n}=22)\end{array}$ & $\begin{array}{l}\text { MCI } \\
(\mathrm{n}=25)\end{array}$ & $\begin{array}{l}\text { AD } \\
(\mathrm{n}=38)\end{array}$ & p value \\
\hline Age, years & $74.7 \pm 4.7$ & $76.0 \pm 5.5$ & $77.4 \pm 4.5$ & 0.119 \\
Education, years & $12.6 \pm 2.1$ & $12.0 \pm 2.8$ & $11.4 \pm 2.1$ & 0.125 \\
Gender, F/M & $12 / 10$ & $15 / 10$ & $26 / 12$ & 0.542 \\
MMSE score & $29.4 \pm 1.2$ & $27.2 \pm 1.5$ & $23.4 \pm 4.1$ & $<0.001$ \\
ADAS score & - & $8.1 \pm 3.5$ & $13.9 \pm 5.0$ & $<0.001$ \\
\hline
\end{tabular}

Mean \pm standard deviation, unless indicated otherwise.

The purpose of this study was to delineate changes in writing ability in patients with the amnestic type of MCI (aMCI) using word and sentence writing tasks and to compare the performance of aMCI patients with that of healthy elderly people and patients with mild AD.

\section{Materials and Methods}

\section{Subjects}

A total of 38 patients with mild AD and 25 patients with aMCI were selected from those admitted to the Neurology Service of Kohnan Hospital for examination. All of the patients were examined by neurologists and received laboratory tests, standard neuropsychological examinations [including the MMSE and the Alzheimer's Disease Assessment Scale Cognitive Subscale (ADAS-Cog)], magnetic resonance imaging of the brain, and magnetic resonance angiography of the neck and head. The ADAS-Cog is a measure of the cognitive domains that are vulnerable in $\mathrm{AD}$, including memory, orientation, visuospatial ability, language, and praxis. Scores range from 0 to 70 , with higher scores reflecting greater cognitive impairment $[18,19]$.

The inclusion criteria for the patients with AD were as follows: (1) a diagnosis of probable AD according to the National Institute of Neurological and Communicative Disorders and Stroke and the Alzheimer's Disease and Related Disorders Association (NINCDS-ADRDA) criteria [20], (2) minimal to mild functional impairment (0.5 and 1 on the Clinical Dementia Rating Scale) [21], (3) right-handedness, and (4) more than 8 years of education. The exclusion criteria were: (1) a history of alcoholism, drug abuse, or other neurological or psychiatric illness, (2) focal brain lesions on MRI, and (3) clinically apparent auditory and/or visual handicaps.

To select patients with aMCI, we adopted the criteria of Petersen et al. [2], which were supplemented by the criteria for aMCI/prodromal AD proposed by Dubois and Albert [22]. The rating scale-based operational criteria included the following: (1) memory complaints corroborated by an informant ( 0.5 for the Clinical Dementia Rating memory box score), (2) progressive onset, (3) memory impairment on neuropsychological assessment relative to healthy age-matched people, (4) typical general cognitive function, (5) largely intact activities of daily living, (6) no clinical dementia, and (7) the exclusion of other disorders that may cause cognitive impairment using adequate tests, including neuroimaging.

To obtain normative writing data for comparison, 22 right-handed healthy subjects were recruited from the community. The control subjects scored 26 or higher on the MMSE.

Written informed consent was obtained from all of the patients and their caregivers and from all of the control subjects prior to their enrollment in the study. The study protocol was approved by the Committee of Medical Ethics, Kohnan Hospital. The demographic data for the $\mathrm{AD}$ group, the aMCI group, and the control group are summarized in table 1. 


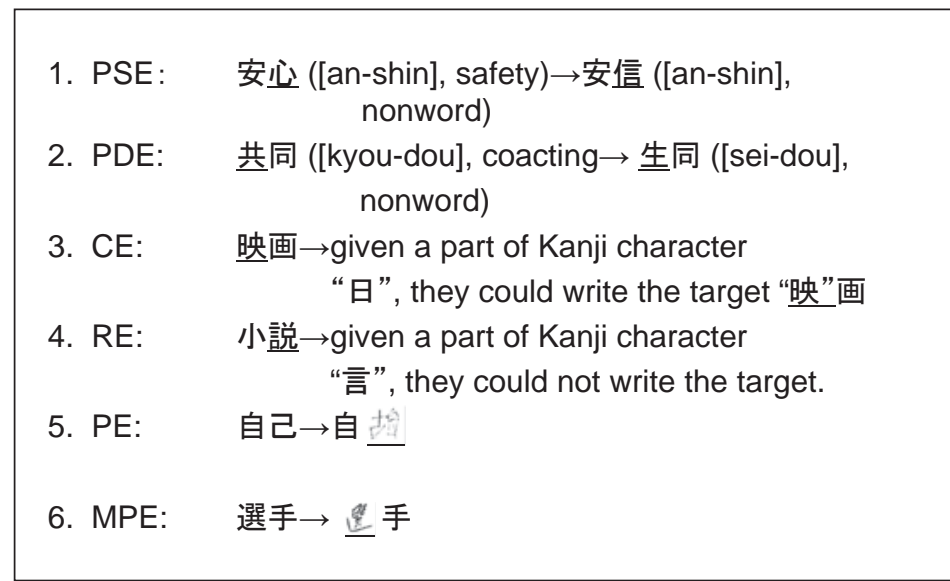

Fig. 1. Samples of the 6 types of writing errors. (1) PSE: phonologically same Kanji error; the target word and the error have the same phonetic value, but the latter is orthographically different from the former and has no meaning as a two-character Kanji word. (2) PDE: phonologically different Kanji error; the target word and the error have different phonetic and orthographic values. When the participant did not respond or stopped after writing one or two strokes, we divided the no response scores into two types of errors, as follows: (3) $\mathrm{CE}$ = cued error; given a part of a target Kanji character, the subject could write the whole character. (4) $\mathrm{RE}$ = recall error; given a part of a Kanji character, the subject could not write the character. (5) PE = peripheral error; an ill-formed or illegible Kanji character. (6) MPE = minor peripheral error; omitting or adding one or two strokes of Kanji.

\section{Task Procedures}

Four tasks were performed to examine the patients' abilities to write Kanji and Kana words and sentences.

\section{Writing to Dictation of Kanji Words}

Fifty auditory word stimuli were used for this task from our previous study [17]. All of the stimuli were conventional compound words consisting of two Kanji characters; half of the words represented concrete nouns, and the other half represented abstract nouns. The stimulus words were read aloud one by one to the subjects, who were asked to write them. These stimuli were selected from a list generated by Ogawa and Inamura [23] to control for concreteness (concrete: $>5$ on a 7-point rating scale for concreteness; abstract: $<3$ ) and ease of learning (both conditions: $>4.9$ on a 7-point rating scale for ease of learning). The word frequencies and script familiarity were not significantly different between the concrete and abstract words [24, 25]. The maximum possible score was 50 .

We classified the errors in the Kanji dictation task into 6 types (see examples in fig. 1) for each character. Although we used two-character Kanji words as stimuli (50 words), the scoring was performed for each Kanji character. The error types were as follows:

(a) Phonologically same Kanji error: substituting a Kanji character with the same phonetic value but a different orthographic value.

(b) Phonologically different Kanji error: substituting a Kanji character with a different phonetic value and a different orthographic value.

If the participant did not respond or stopped writing one or two strokes, we divided no response into two types of errors, as follows:

(c) Cued error: given part of a target Kanji character, the subject could write the whole character. 

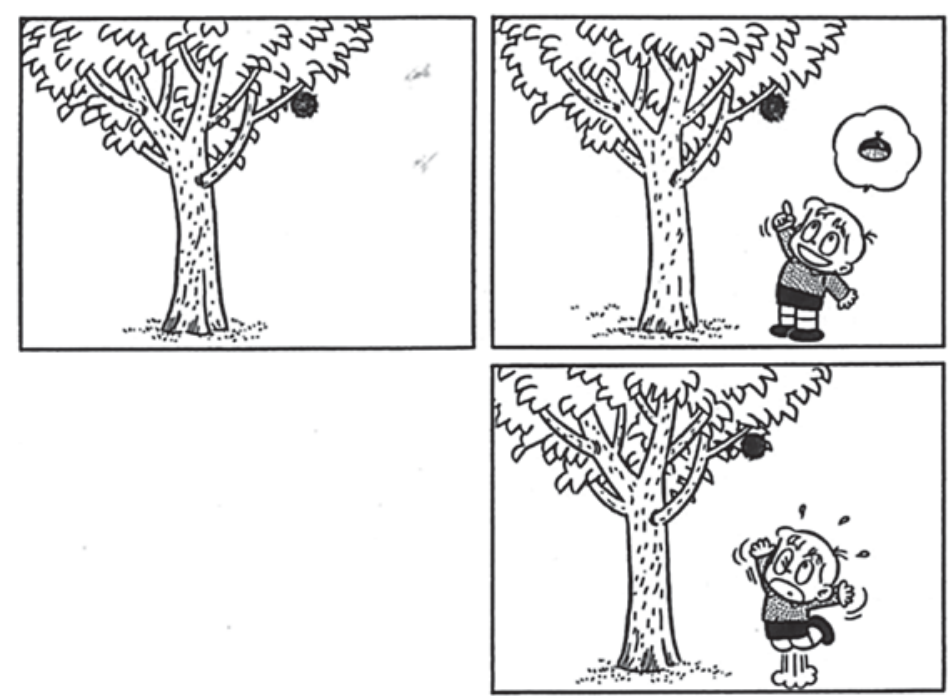

Fig. 2. The picture story (4-frame cartoon) 'a chestnut tree and a child', from the SLTA-ST, was used in the sentence writing task.
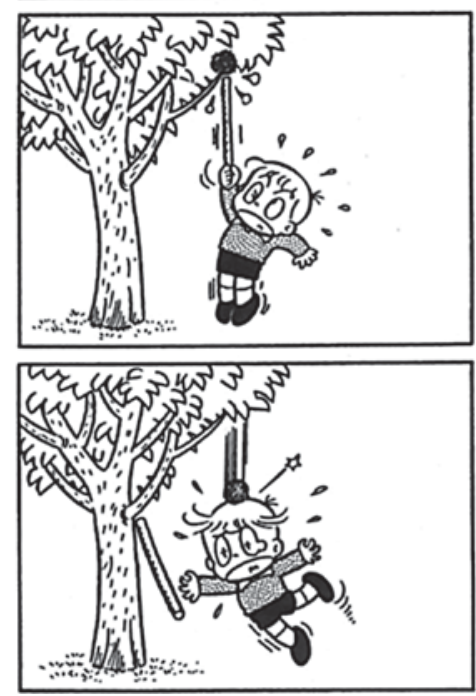

(d) Recall error: given part of a Kanji character, the subject could not write it.

(e) Peripheral error: an ill-formed or illegible Kanji character.

(f) Minor peripheral error: omitting or adding one or two strokes of Kanji.

The maximum error score was 100.

\section{Writing to Dictation of Kana (Hiragana/Katakana) Words}

The words were read aloud one by one to the subjects, who were asked to dictate 10 Hiragana words and 10 Katakana words that were selected from the stimuli in the Kanji writing to dictation task. Because our goal in this task was to examine whether the subjects could or could not write Kana characters compared with their ability to write Kanji characters, we did not consider the script familiarity of the words that used Hiragana or Katakana characters. We selected stimuli containing various types of Kana characters. These Kana words have 2-4 characters. The maximum score was 20 . The performance score had 1 point deducted per word regardless of the number of wrong characters. 
Table 2. Rating of the picture story writing task

\begin{tabular}{llll}
\hline Rating & Number of target words & Syntactical and character error & Smoothing \\
\hline 6 & more than 5 words & 1 error & smooth \\
5 & more than 5 words & 1 or 2 errors & redundant, faltering, recurring \\
4 & 4 or 5 words & 3 or 4 errors & \\
3 & more than 2 words & containing sentence or phrase & \\
2 & more than 1 word & not necessarily contain sentence or phrase & \\
1 & no word & & \\
\hline
\end{tabular}

Seven target words: 'chestnut', 'get', 'stick', 'drop (with stick)', 'head', 'drop (on his head)', and 'ouch'. Syntactic or character error: errors of preposition or termination, Kana or Kanji writing error.

\section{Copying Kanji Words}

The subjects were asked to copy 10 Kanji words that were selected from the Kanji dictation task to examine the difference between writing to dictation and copying. The maximum score was 10 .

\section{Picture Story Writing}

To assess sentence writing abilities, the subjects were asked to give a written description of the picture story (4-frame cartoon) from the Supplementary Test of the Standard Language Test of Aphasia (SLTA-ST). We used the picture of 'a chestnut tree and a child' (fig. 2). That story is more difficult than the SLTA version and represents the middle level of the picture stories in the SLTA-ST. We used this task to evaluate how the subjects understood the stream of a story and to prompt them to increase the number of sentences they wrote.

The task performance was evaluated as follows: a general score from 1 (representing severe deficiency) to 6 (representing normal performance) was generated based on the criteria (SLTA) of the number of content words, grammatical errors, and character errors. The maximum score was 6 . The rating scales are presented in table 2 .

The number of important words related to the picture story [out of the 7 key words: 'chestnut', 'take', 'stick', 'drop (with a stick)', 'head', 'fall onto one's head', and 'ouch!'] that the participant included in his/her writing was calculated. The maximum score was 7.

The errors for each character, word, or sentence were analyzed and classified as lexical or syntactic errors according to the proposed manner [26]. The lexical errors included substitution, omission, letter displacement, incorrect sonant marks, and other types of mistakes for each character. A maximum of one instance per type of error was counted for each character. When there were several errors in a word, the word was counted as including two errors. The errors for the Kanji and Kana characters were classified separately. Variations of Okurigana (supportive Kana characters included after Kanji characters, characteristic of a Japanese reading method) and the selection of Kanji, Hiragana, and Katakana were not penalized. Syntactic errors were deemed to be present if a sentence was incomprehensible, if either a subject or a verb was omitted, if a verb did not correspond to the subject, if one postposition was substituted for another postposition, and if several conjunctions were used within a sentence, resulting in verbosity. When subject-free sentences were repeated and the missing subjects were the same, only the first sentence was counted as an error. When a subject could write words but not a sentence, half of the number of words was counted as errors. 
Table 3. Percentage of word writing performances in the 3 tasks and number (mean \pm standard deviation) of 6 types of Kanji writing errors

\begin{tabular}{lcccc}
\hline & Controls & MCI & AD & p value \\
\hline Kanji words to dictation task & 95.5 & 89.6 & 74.4 & $<0.001$ \\
PSE & $1.0 \pm 1.6$ & $1.9 \pm 2.3$ & $4.8 \pm 3.6$ & $<0.001$ \\
PDE & $0.1 \pm 0.3$ & $0.4 \pm 0.6$ & $1.0 \pm 1.5$ & 0.006 \\
CE & $0.9 \pm 2.2$ & $2.5 \pm 4.6$ & $5.6 \pm 8.6$ & 0.020 \\
RE & $0.2 \pm 0.5$ & $1.0 \pm 2.2$ & $3.6 \pm 5.4$ & 0.003 \\
PE & $0.05 \pm 0.2$ & $0.3 \pm 0.9$ & $1.4 \pm 2.5$ & 0.005 \\
MPE & $0.3 \pm 0.6$ & $1.3 \pm 1.3$ & $1.2 \pm 1.5$ & 0.016 \\
Kana words to dictation task & 99.8 & 98.8 & 98.4 & 0.292 \\
Kanji copying task & 100 & 99.2 & 97.9 & 0.073 \\
\hline
\end{tabular}

PSE = Phonologically same Kanji error; PDE = phonologically different Kanji error; $\mathrm{CE}=$ cued error; RE = recall error; $\mathrm{PE}$ = peripheral error; $\mathrm{MPE}=$ minor peripheral error.

\section{Statistical Analysis}

Statistical analyses were performed using SPSS 22.0 (IBM SPSS Statistics). Demographic data were compared between groups using one-way analyses of variance or $\chi^{2}$ tests. The group effects on the writing task scores were examined using one-way analyses of variance, and post hoc Tukey tests were applied when needed. The significance level was set at $\mathrm{p}<$ 0.05 .

\section{Results}

Age, years of education, and sex were comparable among the groups [table 1 ; for age, $\mathrm{F}(2$, $82)=2.181, p=0.119$; for education, $F(2,82)=2.136, p=0.125$; for $\operatorname{sex}, \chi^{2}(2)=1.226, p=$ 0.542]. The mean MMSE score was significantly lower in the AD group than in the aMCI group and the control group ( $\mathrm{p}<0.001)$, and the score of the aMCI group was lower than that of the control group ( $\mathrm{p}=0.031$ ). The mean ADAS-cog score was significantly higher in the AD group than in the aMCI group $(\mathrm{p}<0.001)$.

Table 3 summarizes the performances in the three word writing tasks. Significant group differences were observed for the Kanji writing task $[\mathrm{F}(2,82)=13.408, \mathrm{p}<0.001]$ but not for the Kana dictation and Kanji copying tasks $[\mathrm{F}(2,82)=1.250, \mathrm{p}=0.292 ; \mathrm{F}(2,82)=2.696, \mathrm{p}=$ 0.073]. The scores on the Kanji writing task were significantly lower for the AD group than for the aMCI ( $p=0.001)$ and control groups $(\mathrm{p}<0.001)$, but there was no significant difference between the aMCI and control groups ( $\mathrm{p}=0.445)$. On the Kana writing task, 31 out of 38 AD patients and 21 out of 25 aMCI patients achieved the full score, and the remainder scored higher than $80 \%$. No patient showed selective impairment on the Kana writing tasks.

Table 3 also describes the number of writing errors on the Kanji dictation task. The group effects for total errors and each type of writing errors were significant [for phonologically same Kanji errors, $\mathrm{F}(2,82)=14.881$, $\mathrm{p}<0.001$; for phonologically different Kanji errors, $F(2,82)=5.468, p=0.006$; for cued errors, $F(2,82)=4.128, p=0.020$; for recall errors, $F(2,82)=6.341, p=0.003$; for peripheral errors, $F(2,82)=5.649, p=0.005$; for minor peripheral errors, $\mathrm{F}(2,82)=4.338, \mathrm{p}=0.016]$. Cued errors were the most common type of errors, followed by phonologically same Kanji errors and recall errors. There were very few phonologically different Kanji errors, peripheral errors, and minor peripheral errors. For phonologically same Kanji errors, recall errors, and peripheral errors, the AD patients 
Dementia

and Geriatric

Cognitive Disorders

Table 4. Number of writing performances on the picture story writing task

\begin{tabular}{|c|c|c|c|c|}
\hline \multicolumn{5}{|c|}{ Dement Geriatr Cogn Disord Extra 2015;5:309-319 } \\
\hline DOI: $10.1159 / 000437297$ & \multicolumn{4}{|c|}{$\begin{array}{l}\text { (c) } 2015 \text { S. Karger AG, Basel } \\
\text { www.karger.com/dee }\end{array}$} \\
\hline \multicolumn{5}{|c|}{$\begin{array}{l}\text { Hayashi et al.: Writing Impairments in Japanese Patients with Mild Cognitive } \\
\text { Impairment and with Mild Alzheimer's Disease }\end{array}$} \\
\hline & Controls & MCI & $\mathrm{AD}$ & $\begin{array}{l}\mathrm{p} \\
\text { value }\end{array}$ \\
\hline Total evaluation score & $5.0 \pm 0.9$ & $4.4 \pm 0.9$ & $4.2 \pm 0.7$ & 0.001 \\
\hline Amount of information & $5.8 \pm 1.2$ & $5.6 \pm 1.5$ & $4.9 \pm 1.2$ & 0.028 \\
\hline Lexical errors & $0.9 \pm 0.9$ & $1.1 \pm 1.4$ & $2.3 \pm 2.0$ & 0.001 \\
\hline Kana errors & $0.7 \pm 0.7$ & $0.6 \pm 0.9$ & $1.6 \pm 1.7$ & 0.006 \\
\hline Excessive character & $0.1 \pm 0.3$ & $0 \pm 0$ & $0.2 \pm 0.6$ & 0.114 \\
\hline Omission & $0.3 \pm 0.6$ & $0.4 \pm 0.8$ & $0.5 \pm 0.8$ & 0.705 \\
\hline Substitution & $0.1 \pm 0.3$ & $0.1 \pm 0.3$ & $0.4 \pm 0.6$ & 0.094 \\
\hline Voiced sound mark & $0.2 \pm 0.4$ & $0.1 \pm 0.3$ & $0.4 \pm 0.9$ & 0.082 \\
\hline Peripheral error & $0 \pm 0$ & $0 \pm 0$ & $0.1 \pm 0.3$ & 0.06 \\
\hline Kanji errors & $0.2 \pm 0.4$ & $0.5 \pm 0.9$ & $0.7 \pm 1.1$ & 0.066 \\
\hline Peripheral error & $0.05 \pm 0.2$ & $0.2 \pm 0.7$ & $0.4 \pm 0.9$ & 0.158 \\
\hline PSE & $0.05 \pm 0.2$ & $0.2 \pm 0.6$ & $0.2 \pm 0.5$ & 0.499 \\
\hline Substitution & $0.1 \pm 0.3$ & $0.1 \pm 0.2$ & $0.1 \pm 0.4$ & 0.510 \\
\hline Syntactic errors & $0.5 \pm 0.8$ & $0.8 \pm 0.9$ & $0.9 \pm 0.7$ & 0.149 \\
\hline
\end{tabular}

Mean \pm standard deviation. PSE $=$ Phonologically same Kanji error.

showed significantly more errors compared with the aMCI group (for phonologically same Kanji errors, $p<0.001$; for recall errors, $p=0.034$; for peripheral errors, $p=0.032$ ) and the control group (for phonologically same Kanji errors, $\mathrm{p}<0.001$; for recall errors, $\mathrm{p}=0.004$; for peripheral errors, $p=0.01$ ), and there were no differences between the aMCI and control groups ( $p>0.1$ for all). For phonologically different errors and cued errors, there were significant differences between the $\mathrm{AD}$ and control groups (for phonologically different errors, $p=0.007$; for cued errors, $p=0.021$ ), and there were no significant differences between the $\mathrm{AD}$ and $\mathrm{aMCI}$ groups or between the aMCI and control groups. For minor peripheral errors, there was no significant difference between the $\mathrm{AD}$ and $\mathrm{aMCI}$ groups, but both groups differed significantly from the control group $(p=0.033$ and $p=0.024$, respectively).

Table 4 shows the performances on the picture story writing task, which was completed by 35 out of $38 \mathrm{AD}$ patients. For the general scores, the amount of information, and the lexical errors on the picture story writing task, the group differences were significant $[\mathrm{F}(2,79)=$ $7.087, p=0.001 ; F(2,79)=3.746, p=0.028 ; F(2,79)=7.176, p=0.001]$; however, there were no significant differences among groups for syntactic errors $[F(2,79)=1.950, p=0.149]$. Regarding lexical errors, the group effect for Kana errors was significant $[F(2,79)=5.526$, $\mathrm{p}=0.006$ ], though not all subtypes of Kana errors were significant [for excessive character, $\mathrm{F}(2,79)=2.233, \mathrm{p}=0.114$; for omission, $\mathrm{F}(2,79)=0.351, \mathrm{p}=0.705$; for substitution, $\mathrm{F}(2$, $79)=2.437, p=0.094$; for voiced sound mark, $F(2,79)=2.579, p=0.082$; for peripheral errors, $F(2,79)=0.666, p=0.06]$. The group effect for Kanji errors and all subtypes of Kanji errors were not significant [for Kanji errors, $F(2,79)=2.815, p=0.066$; for peripheral errors, $F(2,79)=1.888, p=0.158$; for phonologically same Kanji errors, $F(2,79)=0.701, p=0.499$; for substitution, $\mathrm{F}(2,79)=0.678, \mathrm{p}=0.510]$. Regarding the total writing scores, both the AD and aMCI groups scored lower than the control group ( $p=0.001 ; p=0.032)$, but there was no significant difference between the AD and $\mathrm{aMCI}$ groups $(\mathrm{p}=0.58)$. The AD group's writings presented a significantly lower amount of information compared with the control group $(\mathrm{p}=$ $0.041)$, but there were no differences between the AD and aMCI groups ( $p=0.103$ ) or between the aMCI and control groups $(\mathrm{p}=0.895)$. The AD group showed significantly higher lexical error scores compared with the aMCI and control groups ( $p=0.011 ; p=0.004)$, but the aMCI 
Hayashi et al.: Writing Impairments in Japanese Patients with Mild Cognitive Impairment and with Mild Alzheimer's Disease

group's scores did not differ from those of the control group $(p=0.888)$. Regarding lexical errors, there were significant differences in Kana errors between the AD and control groups and between the $\mathrm{AD}$ and $\mathrm{aMCI}$ groups $(\mathrm{p}=0.03 ; \mathrm{p}=0.012)$. There were no differences between the $\mathrm{aMCI}$ and control groups $(\mathrm{p}=0.975)$.

\section{Discussion}

We found that the aMCI patients showed impairments in the picture story writing task, but they had no impairments in the writing or word copying tasks. The patients with mild AD had impairments in writing Kanji words to dictation and in narrative writing but no impairments in writing Kana words or copying Kanji words. The analysis of the error types in the Kanji writing to dictation task demonstrated that the patients with mild AD showed greater impairment than the controls in terms of all types of errors; the most common errors among the AD patients were cued errors, followed by phonologically same Kanji errors and recall errors. The AD patients showed more writing errors, such as phonologically same Kanji errors, recall errors, and peripheral errors, than the aMCI patients. These types of errors could be used to differentiate AD patients from aMCI patients. We confirmed that the writing Kanji words to dictation task was useful for detecting the writing deficits of patients with mild $\mathrm{AD}$ and that the sentence writing tasks allowed to differentiate the writing impairments of aMCI patients from those of controls.

In the present study, the writing of words was preserved in the aMCI patients, which is consistent with the findings of a previous study [4]. By contrast, the mild AD patients demonstrated marked writing difficulties; their most common errors were cued errors, followed by phonologically same Kanji errors and recall errors. The high prevalence of nonresponse errors (cued errors and recall errors) in Kanji writing among the mild AD patients likely resulted from the characters' complexity compared with that of Kana writing or alphabets. In the case of cued errors, the mild AD patients could use part of the Kanji characters as a cue when writing them, even if they could recall the whole characters. This type of error might reflect mild difficulties with recalling the forms of Kanji characters. By contrast, recall errors were more common among the AD patients than among the aMCI patients. This type of error reflects difficulties with whole-character retrieval, and the frequency of these errors might be used to differentiate the writing abilities of AD patients from those of aMCI patients.

Similar to the phonologically plausible errors observed in the context of orthographically irregular words taken from Western alphabets, phonologically same Kanji errors in Kanji writing were common among our AD patients, likely because they had a preserved phonological system but an impaired lexical-semantic system [27]. Phonologically same Kanji errors are classified into a pattern of surface agraphia, which is thought to be equivalent to phonologically plausible errors in Western languages [28]. Phonologically same Kanji errors also allowed to detect the differences in writing abilities between $\mathrm{AD}$ and $\mathrm{aMCI}$ patients.

Our aMCI patients demonstrated more minor peripheral errors than the controls, although other types of errors made by the aMCI patients did not differ significantly from those of the controls. Despite the absence of a significant difference between the aMCI patients and the mild $\mathrm{AD}$ patients in the number of minor peripheral errors, the number of other types of errors was significantly higher in the mild AD group. Minor peripheral errors might not reflect Kanji writing abilities themselves but other cognitive problems in aMCI, such as attentional deficits. Because of the effect of the small sample size in our study, however, the power of statistics was reduced. A difference between the control group and the aMCI patient group in the Kanji writing task may well emerge if the sample size is larger. 
Hayashi et al.: Writing Impairments in Japanese Patients with Mild Cognitive Impairment and with Mild Alzheimer's Disease

The mild AD patients showed writing impairments in the general scores for picture story writing, as a previous study found [8]. The majority of previous studies show a decreased amount of information [6,9-13] and more lexical errors among mild AD patients compared with controls $[6,8,11,15]$, consistent with our findings. In the present study, the number of Kana errors was much higher than the number of Kanji errors, contrary to the results of the word writing to dictation tasks. When the AD patients could not recall the Kanji characters, they tended to write Kana characters instead, which made the Kanji errors discreet. Lexical difficulty is a feature of mild AD that does not appear in aMCI. In contrast with the abovementioned errors, the number of syntactic errors was not higher among the mild AD patients. Although AD patients reportedly write grammatically simpler sentences $[10,12,13]$, studies have also suggested that the syntactic rule system is preserved even in the later stages of $\mathrm{AD}[13]$.

Only the general score for the picture story writing task significantly deteriorated in the aMCI patients. Although few studies have addressed the general evaluation of writing impairments, some studies have demonstrated slight factorial writing difficulties in patients with minimal AD $[12,13,15]$. These studies suggest that minimal AD patients appear to have difficulties with semantic processing or cognitive decision-making in addition to writing. Our aMCI patients might show impairments in the general evaluation as the sum of the individual factor evaluations. Their impairments might be affected by slight cognitive impairments beyond writing difficulties themselves, such as attention or semantic memory impairments. Written description appears to be a more sensitive test of semantic or lexical difficulties in mild AD and is undeniably a more reliable test for detecting the subtle degradation of multicomponent cognitive functions in patients with aMCI.

In conclusion, we found that the patients with mild AD showed difficulty in writing Kanji characters with preserved Kana writing ability, and the aMCI patients showed impairments in the writing picture story task with preserved word writing ability. The Kanji writing to dictation task manifested the writing impairments in mild AD patients. The picture story writing task demands the complex integration of multiple cognitive functions; therefore, our findings suggest that sentence writing could be used to sensitively detect not elemental writing deficits, but complex integrated cognitive abilities in aMCI patients.

\section{Acknowledgements}

This study was partly supported by a Grant-in-Aid for Science Research (C) to A. Hayashi (23530946), the Global COE Program (Basic and Translational Research Center for Global Brain Science), MEXT, Japan, Health and Labour Sciences Research Grants (Research on Dementia). We express our thanks to the patients and controls who participated in this study.

\section{Disclosure Statement}

We declare that we have no conflicts of interest. 
Hayashi et al.: Writing Impairments in Japanese Patients with Mild Cognitive Impairment and with Mild Alzheimer's Disease

\section{References}

1 Adlam AL, Bozeat S, Arnold R, Watson P, Hodges JR: Semantic knowledge in mild cognitive impairment and mild Alzheimer's disease. Cortex 2006;42:675-684.

-2 Petersen RC, Smith GE, Waring SC, Ivnik RJ, Tangalos EG, Kokmen E: Mild cognitive impairment: clinical characterization and outcome. Arch Neurol 1999;56:303-308.

-3 Ribeiro F, de Mendonca A, Guerreiro M: Mild cognitive impairment: deficits in cognitive domains other than memory. Dement Geriatr Cogn Disord 2006;21:284-290.

4 Hughes JC, Graham N, Patterson K, Hodges JR: Dysgraphia in mild dementia of Alzheimer's type. Neuropsychologia 1997;35:533-545.

5 Croisile B: Agraphia in Alzheimer's disease. Dement Geriatr Cogn Disord 1999;10:226-230.

-6 Henderson VW, Buckwalter JG, Sobel E, Freed DM, Diz MM: The agraphia of Alzheimer's disease. Neurology 1992;42:777-784.

7 Rapcsak SZ, Arthur SA, Bliklen DA, Rubens AB: Lexical agraphia in Alzheimer's disease. Arch Neurol 1989;46: 65-68.

8 Horner J, Heyman A, Dawson D, Rogers H: The relationship of agraphia to the severity of dementia in Alzheimer's disease. Arch Neurol 1988;45:760-763.

9 Croisile B, Carmoi T, Adeleine P, Trillet M: Spelling in Alzheimer's disease. Behav Neurol 1995;8:135-143.

10 Croisile B, Ska B, Brabant MJ, Duchene A, Lepage Y, Aimard G, Trillet M: Comparative study of oral and written picture description in patients with Alzheimer's disease. Brain Lang 1996;53:1-19.

11 Neils J, Boller F, Gerdeman B, Cole M: Descriptive writing abilities in Alzheimer's disease. J Clin Exp Neuropsychol 1989;11:692-698.

12 Forbes KE, Shanks MF, Venneri A: The evolution of dysgraphia in Alzheimer's disease. Brain Res Bull 2004;63: 19-24.

$\checkmark 13$ Kemper S, LaBarge E, Ferraro FR, Cheung H, Storandt M: On the preservation of syntax in Alzheimer's disease. Evidence from written sentences. Arch Neurol 1993;50:81-86.

$\checkmark 14$ Kumar V, Giacobini E: Use of agraphia in subtyping of Alzheimer's disease. Arch Gerontol Geriatr 1990;11: 155-159.

15 LaBarge E, Smith DS, Dick L, Storandt M: Agraphia in dementia of the Alzheimer type. Arch Neurol 1992;49: 1151-1156.

16 Soma Y, Sugishita M, Kitamura K, Maruyama S, Imanaga H: Lexical agraphia in the Japanese language. Pure agraphia for kanji due to left posteroinferior temporal lesions. Brain 1989;112:1549-1561.

17 Hayashi A, Nomura H, Mochizuki R, Ohnuma A, Kimpara T, Ootomo K, Hosokai Y, Ishioka T, Suzuki K, Mori E: Neural substrates for writing impairments in Japanese patients with mild Alzheimer's disease: a SPECT study. Neuropsychologia 2011;49:1962-1968.

-18 Mohs RC, Rosen WG, Davis KL: The Alzheimer's disease assessment scale: an instrument for assessing treatment efficacy. Psychopharmacol Bull 1983;19:448-450.

19 Honma A, Fukuzawa K, Tsukada Y, Ishii T, Hasegawa, K, Mohs RC: Development of a Japanese version of Alzheimer's Disease Assessment Scale (ADAS). Jpn J Geriatr Psychiatry 1992;3:647-655.

-20 McKhann G, Drachman D, Folstein M, Katzman R, Price D, Stadlan EM: Clinical diagnosis of Alzheimer's disease: report of the NINCDS-ADRDA work group under the auspices of department of health and human services task force on Alzheimer's disease. Neurology 1984;34:939-944.

21 Morris JC: The Clinical Dementia Rating (CDR): current version and scoring rules. Neurology 1993;43:24122414.

22 Dubois B, Albert ML: Amnestic MCI or prodromal Alzheimer's disease? Lancet Neurol 2004;3:246-248.

23 Ogawa T, Inamura Y: An analysis of word attributes, imagery, concreteness, meaningfulness and ease of learning for Japanese nouns (in Japanese). Jpn J Psychol 1974;44:317-327.

24 Amano S, Kondo T: NTT Database Series. Nihongo-no Goitokusei: Lexical Properties of Japanese, ed 1. Tokyo, Sanseido, 2000, vol 7.

25 Kondo T, Amano S: NTT Database Series. Nihongo-no Goitokusei: Lexical Properties of Japanese, ed 1. Tokyo, Sanseido, 1999, vol 2.

-26 Tsuji-Akimoto S, Hamada S, Yabe I, Tamura I, Otsuki M, Kobashi S, Sasaki H: Writing errors as a result of frontal dysfunction in Japanese patients with amyotrophic lateral sclerosis. J Neurol 2010;257:2071-2077.

-27 Hayashi A, Suzuki K, Ohigashi Y, Takatsuki Y, Nakano Y, Yamadori A, Mori E: Writing Kanji without semantics in a case with probable Alzheimer's disease. Brain Nerve 2002;54:481-488.

28 Sakurai Y, Tsuchiya K, Oda T, Hori K, Tominaga I, Akiyama H, Bando M, Haga C, Iwata M, Mannen T: Ubiquitinpositive frontotemporal lobar degeneration presenting with progressive Gogi (word-meaning) aphasia. A neuropsychological, radiological and pathological evaluation of a Japanese semantic dementia patient. J Neurol Sci 2006;250:3-9. 